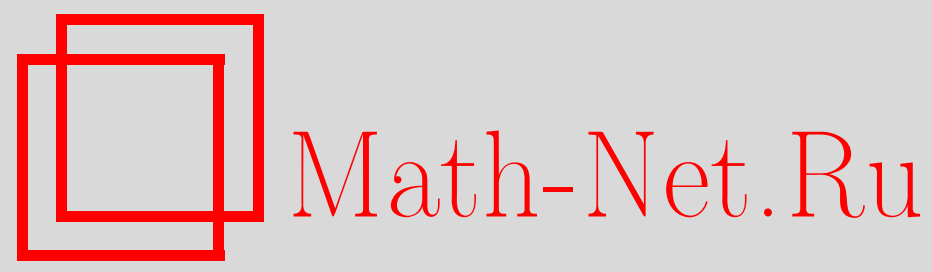

А. С. Рыжов, О реализации умножения полиномиальных матриц над полем $G F(2)$ с помощью быстрого преобразования Фурье, Дискрет. матем., 2013, том 25, выпуск 1, 111120

DOI: https://doi.org/10.4213/dm1224

Использование Общероссийского математического портала Math-Net.Ru подразумевает, что вы прочитали и согласны с пользовательским соглашением http://www. mathnet.ru/rus/agreement

Параметры загрузки:

IP : 54.81 .137 .203

26 апреля 2023 г., 17:35:47

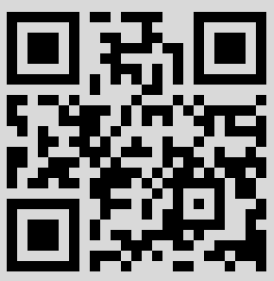




\title{
О реализации умножения полиномиальных матриц над полем $G F(2)$ с помощью быстрого преобразования Фурье
}

\author{
() 2013 г. А. С. Рыжов
}

\begin{abstract}
В статье рассматривается задача реализации быстрого умножения двоичных многочленов и полиномиальных матриц на 64-разрядных ЭВМ. Предложен метод, позволяющий эффективно выполнять быстрое преобразование Фурье с помощью операций сложения и умножения по модулю 264 при ограничении на размер задачи. Приведены результаты экспериментальных сравнений, показывающие существенные преимущества реализации предложенного алгоритма перед функцией умножения двоичных многочленов математической библиотеки NTL при умножении многочленов степени выше 225 и при умножении квадратных полиномиальных матриц степени от 214 и размера от $32 \times 32$.
\end{abstract}

\section{1. Введение}

Умножение полиномиальных матриц является базовой операцией многих алгоритмов. К таковым, например, относится алгоритм Томэ построения векторных аннулирующих многочленов для матричных последовательностей [1], что, в свою очередь, используется при нахождении решений сильно разреженных систем линейных уравнений (см., например, $[2,3])$. Поэтому эффективная реализация процедуры умножения полиномиальных матриц играет важную роль в снижении трудоемкости этих алгоритмов.

При перемножении полиномиальных матриц естественным образом возникает подзадача, связанная с умножением многочленов - элементов этих матриц. Стандартный алгоритм умножения двух многочленов $f(x), g(x) \in P[x]$ степени не выше $n$ над полем $P$ требует $O\left(n^{2}\right)$ операций умножения (и сложения) в поле $P$. Существует большое количество алгоритмов, позволяющих реализовать данную задачу более эффективно, то есть с трудоемкостью, меньшей $O\left(n^{2}\right)$ операций умножения в поле $P$. Классическим примером здесь является рекурсивный алгоритм Карацубы (см., например, [4]), сводящий умножение многочленов степени $2^{t}$ к трем умножениям многочленов степени $2^{t-1}$; трудоемкость этого алгоритма равна $O\left(n^{\log _{2} 3}\right)$ операций в поле. При разбиении многочленов на большее число частей можно добиться снижения показателя: для любого $\varepsilon>0$ существует алгоритм умножения со сложностью $O\left(n^{1+\varepsilon}\right)$ (метод Тоома-Кука).

В 1971 г. Шёнхаге и Штрассен предложили новый способ умножения целых чисел, основанный на использовании быстрого преобразования Фурье; трудоемкость метода равна $O(n \log n)$ арифметических операций или $O(n \log n \log \log n)$ двоичных операций, где $n$ 
- количество цифр в двоичной записи произведения (см. [5, 6]). Метод также применим для умножения многочленов над любыми полями характеристики, отличной от 2. Кроме того, авторы метода высказали гипотезу относительно нижней границы трудоемкости умножения чисел: $O(n \log n)$ двоичных операций. В 2007 г. швейцарский математик Мартин Фурье предложил асимптотически более быстрый алгоритм умножения чисел с трудоемкостью $\left.n \log n 2^{O\left(\mathrm{~g}^{*} n\right.}\right)$ где $\lg ^{*} n-$ так называемый итеративный логарифм:

$$
\lg ^{*} n=\min \left\{i \geqslant 0: \lg ^{(i)} n \leqslant 1\right\} \quad \lg ^{(0)} n=n, \quad \lg ^{(i+1)} n=\lg \lg ^{(i)} n,
$$

однако на практике выигрыш данного алгоритма (по сравнению с алгоритмом ШёнхагеШтрассена) достигается лишь на астрономически больших числах (см. [7]).

Отметим также, что на практике метод Шёнхаге-Штрассена начинает превосходить более ранние методы (такие, как метод Карацубы), начиная с целых чисел длины, большей $2^{17}$ двоичных знаков; на меньших размерах задачи алгоритм Карацубы оказывается эффективнее, а для совсем небольших чисел (до 128 битов) наиболее быстрым является умножение с помощью заранее вычисленных таблиц.

В данной статье рассматривается задача реализации быстрого умножения многочленов над полем $G F(2)$ для перемножения двоичных полиномиальных матриц. Предложен метод эффективной реализации быстрого преобразования Фурье для умножения двоичных многочленов с помощью арифметики в кольце вычетов по простому модулю при ограничении на степень преобразования. Приведены результаты сравнения времени работы предложенной реализации и функции умножения многочленов, реализованной в известной математической библиотеке NTL. Показано, что реализация предложенного метода работает быстрее функции умножения двоичных многочленов библиотеки NTL при степенях многочленов выше $2^{25}$. В случае, если сравниваемые функции используются для умножения полиномиальных матриц, предложенный метод эффективнее уже для матриц размера $32 \times 32$ и степени больше $2^{14}$. Заметим, что при нахождении решений системы линейных уравнений для факторизации 768-битного числа (см. [3]) на одном из этапов потребовалось умножение двух полиномиальных матриц размера $1536 \times 1536$ и степени $565000 \approx 2^{19}$.

\section{2. Умножение многочленов с помощью быстрого преобразования Фурье}

Приведем описание метода умножения многочленов с помощью быстрого преобразования Фурье, следуя, в основном, изложению [8], п. 4.3. Итак, пусть $f(x)$ и $g(x)-$ два многочлена над некоторым полем $P$,

$$
f(x)=\sum_{i=0}^{n-1} f_{i} x^{i}, \quad g(x)=\sum_{i=0}^{n-1} g_{i} x^{i} .
$$

Если обозначить $f_{i}=g_{i}=0$ при $i \geqslant n$, то коэффициенты произведения

$$
h(x)=f(x) g(x)=\sum_{i=0}^{2 n-2} h_{i} x^{i}
$$

выражаются в виде свертки:

$$
h_{i}=f_{0} g_{i}+f_{1} g_{i-1}+\ldots+f_{i-1} g_{1}+f_{i} g_{0} .
$$


Пусть $\omega-$ некоторый элемент поля $P$ с мультипликативным порядком ord $\omega=K>2 n-2$. Если размер поля $P$ меньше $2 n$, то необходимо выбрать его алгебраическое расширение и в нем выполнять все операции. Дискретное преобразование Фурье (ДПФ) последовательности $F=\left(f_{0}, \ldots, f_{K-1}\right)$ можно определить как последовательность $\widehat{F}=\left(\hat{f}_{0}, \ldots, \hat{f}_{K-1}\right)$ где

$$
\hat{f_{s}}=\sum_{j=0}^{K-1} \omega^{s j} f_{j} .
$$

Если $\left(\hat{g}_{0}, \ldots, \hat{g}_{K-1}\right)$ - аналогичное преобразование для $\left(g_{0}, \ldots, g_{K-1}\right)$ то, очевидно, $\left(\hat{f}_{0} \hat{g}_{0}, \ldots, \hat{f}_{K-1} \hat{g}_{K-1}\right)$ есть ДПФ для $\left(h_{0}, \ldots, h_{K-1}\right)$. Следовательно, если существует эффективный алгоритм вычисления ДПФ и обратного ДПФ, то произведение многочленов $h(x)=f(x) g(x)$ можно вычислять следующим образом:

(1) Вычислить преобразования Фурье $\left(\hat{f}_{0}, \ldots, \hat{f}_{K-1}\right)$ и $\left(\hat{g}_{0}, \ldots, \hat{g}_{K-1}\right)$ для последовательностей коэффициентов $\left(f_{0}, \ldots, f_{K-1}\right)$ и $\left(g_{0}, \ldots, g_{K-1}\right)$.

(2) Вычислить последовательность $\left(\hat{h}_{0}, \ldots, \hat{h}_{K-1}\right)$, где $\hat{h}_{i}=\hat{f}_{i} \hat{g}_{i}$.

(3) Вычислить обратное преобразование Фурье $\left(h_{0}, \ldots, h_{K-1}\right)$ для последовательности $\left(\hat{h}_{0}, \ldots, \hat{h}_{K-1}\right)$. Если $\operatorname{deg} f(x), \operatorname{deg} g(x)<n, K \geqslant 2 n-1$, то

$$
f(x) g(x)=\sum_{i=0}^{2 n-2} h_{i} x^{i} .
$$

Основную трудоемкость данного алгоритма составляет вычисление ДПФ двух последовательностей длины $K$ и обратного ДПФ порядка $K$, поскольку покоординатное умножение двух векторов ДПФ выполняется с линейной сложностью. Для лобового вычисления ДПФ (иными словами, по формуле) последовательности длины $K$ требуется $O\left(K^{2}\right)$ операций (умножения) в поле $P$. Быстрое преобразование Фурье (БПФ) - это алгоритм, который вычисляет дискретное преобразование Фурье асимптотически быстрее, чем $O\left(K^{2}\right)$ операций в поле. Если число $K$ является степенью 2, то ДПФ может быть вычислено с трудоемкостью порядка $O(K \log K)$ операций в поле; соответствующий алгоритм называют быстрым преобразованием Фурье (БПФ). Для последовательности $\left(u_{0}, \ldots, u_{K-1}\right)$ длины $K=2^{k}$ ДПФ $\left(\hat{u}_{0}, \ldots, \hat{u}_{K-1}\right)$ порядка $K$ с элементом $\omega$, $\operatorname{ord} \omega=K$, можно вычислить по следующей схеме.

Шаг 0. Для $t=0,1, \ldots, K-1$ присвоить $A_{t_{k-1}, \ldots, t_{0}}^{0}:=u_{t}$, где

$$
t=\sum_{i=0}^{k-1} t_{i} 2^{i}
$$

Шаг 1. Присвоить

$$
A_{s_{k-1}, t_{k-2}, \ldots, t_{0}}^{1}:=A_{0, t_{k-2}, \ldots, t_{0}}^{0}+\omega^{2^{k-1} s_{k-1}} A_{1, t_{k-2}, \ldots, t_{0}}^{0} .
$$

Шаг 2. Присвоить

$$
A_{s_{k-1}, s_{k-2}, t_{k-3}, \ldots, t_{0}}^{2}:=A_{s_{k-1}, 0, t_{k-3}, \ldots, t_{0}}^{1}+\omega^{2^{k-2} s_{k-1}+2^{k-1} s_{k-2}} A_{s_{k-1}, 1, t_{k-3}, \ldots, t_{0}}^{1} .
$$


$\ldots$

Шаг $k$. Присвоить

$$
A_{s_{k-1}, \ldots, s_{1}, s_{0}}^{k}:=A_{s_{k-1}, \ldots, s_{1}, 0}^{k-1}+\omega^{s_{k-1}+2 s_{k-2}+\ldots+2^{k-1} s_{0}} A_{s_{k-1}, \ldots, s_{1}, 1}^{k-11} .
$$

Для

$$
s=\sum_{i=0}^{k-1} s_{i} 2^{i}
$$

соответствующий элемент ДПФ равен $\hat{u}_{s}=A_{s_{0}, s_{1}, \ldots, s_{k-1}}^{k}$ (см. [8], п. 4.3). На каждом шаге набор $\left(s_{k-1}, \ldots, t_{0}\right)$ пробегает все $K=2^{k}$ возможные значения, поэтому трудоемкость каждого шага есть $O\left(2^{k}\right)$ операций в поле. Следовательно, трудоемкость алгоритма есть $O\left(k 2^{k}\right)$, то есть $O(K \log K)$ операций в поле $P$. При многократном применении алгоритма БПФ элементы $\omega^{i}, i=1,2, \ldots, K-1$, можно вычислить на предварительном этапе.

Заметим, что двойное преобразование Фурье имеет вид

$$
\hat{\hat{u}}_{i}=K u_{(-i) \bmod K},
$$

поэтому обратное преобразование Фурье можно получить так же, как и прямое, разделив затем на $K$ и выполнив соответствующую перестановку. Заметим, что деление на $K$ возможно, поскольку характеристика поля char $P$ не делит порядок элемента поля $\operatorname{ord} \omega=K$.

Данный метод был предложен Штрассеном в 1968 г. для быстрого умножения больших чисел. Позже Штрассен и Шёнхаге уточнили данный метод, а также распространили его на умножение многочленов над полем характеристики, отличной от 2 (см. [5, 6]). При этом в качестве элемента $\omega$ предлагалось использовать комплексный корень $K$-й степени из 1 , именно, $\omega=e^{2 \pi i / K} \in \mathbf{C}$. Похожие идеи были (независимо) предложены Поллардом в [9].

Алгоритм быстрого преобразования Фурье можно сформулировать с помощью рекурсии. В этом случае основной шаг состоит в сведении задачи для $K$ элементов к задаче для $q=K / p$ элементов, где $p$ есть делитель $K$. Заметим, что

$$
\hat{u}_{i}=\sum_{s=0}^{K-1} \omega^{i s} u_{s}=\sum_{j=0}^{p-1} \omega^{i j}\left(\sum_{t=0}^{q-1} \omega^{i t p} u_{j+t p}\right) .
$$

Нетрудно видеть, что при фиксированном значении $j$ сумма в скобках есть $i$-й элемент ДПФ порядка $q$ для последовательности $\left(u_{j}, u_{j+p}, u_{j+2 p}, \ldots, u_{j+t p}, \ldots, u_{j+(q-1) p}\right)$. Если эти ДПФ уже вычислены, то для каждого $i \in\{0, \ldots, K-1\}$ трудоемкость вычисления $\hat{u}_{i}$ равна $O(p)$ операций, а для всех $\hat{u}_{i}$ она равна $O(K p)$. По индукции легко доказать, что если $K=p_{1} \cdots p_{s}$, то трудоемкость приведенного алгоритма равна $O\left(K\left(p_{1}+\ldots+p_{s}\right)\right)$. В частности, при $K=2^{k}$ трудоемкость равна $O(K \log K)$.

В расширении полей характеристики 2 не существует элементов порядка $2^{k}$, поэтому оптимальная трудоемкость в случае $P=G F(2)$ недостижима. Тем не менее, можно подобрать поле расширения, в котором будут существовать элементы, порядок которых состоит из произведения многих маленьких сомножителей. Например, в поле $G F\left(2^{60}\right)$ с мультипликативной группой мощности

$$
\left|P^{*}\right|=2^{60}-1=3^{2} \cdot 5^{2} \cdot 7 \cdot 11 \cdot 13 \cdot 31 \cdot 41 \cdot 61 \cdot 151 \cdot 331 \cdot 1321
$$


можно выбрать элемент порядка

$$
K=3^{2} \cdot 5^{2} \cdot 7 \cdot 11 \cdot 13=225225
$$

что позволяет реализовать умножение многочленов степени не выше 112612. Вопросы, связанные с умножением многочленов над полем характеристики 2, рассмотрены в работе Шёнхаге [10] (см. также [6], теорема 2.13, пример 2.6).

При перемножении многочленов над полем $P=G F(2)$ можно воспользоваться одним из следующих способов.

- Перемножать многочлены в кольце целых чисел, выполняя БПФ в поле комплексных чисел с элементом $\omega=e^{2 \pi i / K} \in \mathbf{C}$. После перемножения редуцировать коэффициенты результата до $P=\mathbf{Z}_{2}=G F(2)$.

- Использовать алгебраическое расширение поля $P=G F(2)$ с мультипликативной группой, порядок которой содержит большое число маленьких делителей, как в приведенном выше примере с полем $G F\left(2^{60}\right)$.

- Для $K=3^{k}$ использовать БПФ порядка $3^{\lfloor k / 2\rfloor}$ в алгебре

$$
P_{3, K}=P[x] /\left(1+x^{K}+x^{2 K}\right),
$$

рекурсивно сводя одну операцию умножения в $A_{3, K}$ к нескольким умножениям в $A_{3,3\lceil k / 2\rceil}$ (см. [6], пример 2.6).

С точки зрения реализации на ЭВМ, каждый из приведенных способов имеет свои недостатки. При классическом подходе, связанном с проведением вычислений в поле комплексных чисел, необходимо учитывать ограниченную точность вычислений, предоставляемую современными процессорами. Данный вопрос подробно рассмотрен в [8], п. 4.3. Реализация в алгебрах $G F\left(2^{k}\right)$ или $G F(2)_{3,3^{k}}$ исключает потерю точности, однако требует большого числа элементарных операций над машинными словами для реализации одной операции умножения. Автором предложен метод, позволяющий быстро вычислять произведение многочленов и их линейных комбинаций, используя целочисленные операции 64-разрядных ЭВМ, при определенных ограничениях на степени исходных многочленов. Метод излагается в следующем разделе и основан на проведении всех вычислений в простом поле $\mathbf{Z}_{N}$, содержащим элемент порядка $2^{k}$, где

$$
2^{k}>\operatorname{deg}(f)+\operatorname{deg}(g)
$$

для любых элементов $f(x), g(x) \in G F(2)[x]$, участвующих в вычислениях.

\section{3. Об одном способе реализации умножения многочленов над полем $G F(2)$}

Приведем один способ эффективной реализации умножения двоичных многочленов небольшой степени. Пусть

$$
f(x)=\sum_{i} f_{i} x^{i}, \quad g(x)=\sum_{j} g_{j} x^{j}
$$


- два многочлена над полем $P=G F(2)$. Коэффициенты произведения

$$
h(x)=f(x) g(x)=\sum_{k} h_{k} x^{k}
$$

представляются в виде свертки

$$
h_{k}=\sum_{i, j: i+j=k} f_{i} g_{j} \bmod 2 ;
$$

здесь операции сложения и умножения выполняются в кольце целых чисел. Если степень произведения ограничена сверху числом $N: \operatorname{deg} h(x)<N$, то, очевидно,

$$
h_{k}=\left(\sum_{i, j: i+j=k} f_{i} g_{j} \bmod N\right) \bmod 2,
$$

поскольку $f_{i} g_{j} \in\{0,1\}$, а $k<N$. Это означает, что найдется произведение многочленов $f(x)$ и $g(x)$, рассматриваемых как многочлены над кольцом вычетов $\mathbf{Z}_{N}$, и остается взять коэффициенты их произведения в $\mathbf{Z}_{N}$ по модулю 2 .

Если число $N$ простое, то для вычисления произведения $f(x) g(x)$ в поле $\mathbf{Z}_{N}$ можно использовать БПФ. Для этого необходимо, чтобы в $\mathbf{Z}_{N}$ был элемент $\omega \in \mathbf{Z}_{N}$ порядка

$$
\operatorname{ord} \omega=K=2^{k}>\operatorname{deg} f(x)+\operatorname{deg} g(x) .
$$

В качестве модуля $N$ можно выбирать простые числа вида $N=2^{k} p+1$, где $p-$ небольшое нечетное число. Например, при

$$
N=3 \cdot 2^{18}+1
$$

в поле $\mathbf{Z}_{N}$ есть элемент порядка $2^{18}$, и данный метод подходит для перемножения многочленов, произведение которых имеет степень не выше $2^{18}$. Для перемножения многочленов степени меньше $n=2^{25}$ можно использовать элемент $\omega$ порядка ord $\omega=2^{26}$ в поле $\mathbf{Z}_{N}$ при

$$
N=7 \cdot 2^{26}+1
$$

В классическом алгоритме БПФ основной операцией на каждом шаге является вычисление элементов вида $a_{0}+\omega^{s} a_{1}$, поэтому при $N<2^{32}$ предложенный метод допускает эффективную реализацию на 64-разрядных ЭВМ: в качестве базовых операций используются сложение и умножение целых чисел по модулю $2^{64}$.

\section{4. Умножение полиномиальных матриц с помощью быстрого преобразования Фурье}

Вернемся к задаче умножения полиномиальных матриц. При классическом алгоритме умножения двух матриц $A=\left(a_{i j}\right)$ и $B=\left(b_{i j}\right)$ элемент произведения $C=\left(c_{i j}\right)=A B$ вычисляется по формуле

$$
c_{i j}=\sum_{s} a_{i s} b_{s j}
$$

Если элементы матриц - многочлены, то с помощью ДПФ можно ускорить процедуру умножения следующим образом: 
- вычислить ДПФ элементов сомножителей: $a_{i j} \rightarrow \hat{a}_{i j}, b_{i j} \rightarrow \hat{b}_{i j}$;

- перемножить матрицы $\hat{A}=\left(\hat{a}_{i j}\right)$ и $\widehat{B}=\left(\hat{b}_{i j}\right)$, состоящие из вычисленных векторов ДПФ, получив в результате матрицу с ДПФ произведения $\widehat{C}=\left(\hat{c}_{i j}\right)$;

- для всех элементов матрицы $\widehat{C}$ выполнить обратное ДПФ и получить результат: $\hat{c}_{i j} \rightarrow c_{i j}$.

При перемножении матриц $\widehat{C}=\hat{A} \widehat{B}$ можно пользоваться стандартным алгоритмом умножения. При этом трудоемкость операций сложения и умножения векторов ДПФ в формуле

$$
\hat{c}_{i j}=\sum_{s} \hat{a}_{i s} \hat{b}_{s j}
$$

пропорциональна порядку ДПФ, то есть линейна относительно степени полиномиальных матриц $A$ и $B$. Если перемножаются матрицы размеров $m \times m$ степеней $\operatorname{deg} A, \operatorname{deg} B<n$, а поле $P$ допускает быстрое преобразование Фурье, то трудоемкость вычисления произведения $C=A B$ с использованием БПФ равна $O\left(m^{2} n \log n+m^{3} n\right)$ операций в поле. Конечно, матрицы не обязательно должны быть квадратными. Данное условие не ограничивает общности и введено исключительно для упрощения изложения. Отметим, что при использовании любого другого алгоритма умножения многочленов, например, метода Карацубы, с трудоемкостью $Q(n)$ операций в $P$, где $n-$ степень многочленов, трудоемкость умножения полиномиальных матриц составит $O\left(m^{3} Q(n)\right)$ операций в $P$. Поскольку $Q(n)>n$ операций в поле $P$, при перемножении матриц достаточно большого размера $m$ алгоритм на основе БПФ эффективнее других алгоритмов.

Разумеется, как для перемножения матриц ДПФ $\hat{A}$ и $\widehat{B}$, так и для перемножения самих полиномиальных матриц $A$ и $B$ с использованием метода умножения многочленов, не основанного на БПФ, можно применять более эффективные алгоритмы, нежели стандартное умножение по определению

$$
c_{i j}=\sum_{s} a_{i s} b_{s j}
$$

Например, предложенный в 1969 г. Штрассеном алгоритм имеет трудоемкость $O\left(m^{\log _{2} 7}\right)$ операций умножения элементов (вместо $O\left(m^{3}\right)=O\left(m^{\log _{2} 8}\right)$, как в стандартном алгоритме). Однако при небольших значениях $m$ стандартный алгоритм работает быстрее за счет меньшей мультипликативной константы в формуле трудоемкости. Также следует отметить одну особенность, связанную с умножением двоичных полиномиальных матриц с помощью БПФ на основе метода, изложенного в предыдущем разделе. Поскольку все вычисления выполняются в поле $\mathbf{Z}_{N}$ (при $N<2^{32}$ ) с помощью машинных операций сложения и умножения по модулю $2^{64}$, необходимо каждый раз выполнять вычисление остатка от деления на $N$, то есть взятия числа по модулю $N$. Эта операция выполняется относительно долго (во много раз дольше, чем сложение и умножение по модулю $2^{64}$ ). Тем не менее, при дополнительных ограничениях на число $N$ можно сократить число выполнений этой операции. Например, если $m N^{2}<2^{64}$, то при вычислении

$$
\hat{c}_{i j}=\sum_{s=1}^{m} \hat{a}_{i s} \hat{b}_{s j}
$$

операцию взятия по модулю $N$ можно выполнить только в самом конце, поскольку каждый элемент вектора ДПФ меньше $N$, а сумма из $m$ произведений таких элементов, 
соответственно, меньше $m N^{2}$, и в результате вычислений не возникает выхода за границу $2^{64}$. В общем случае, если $t N^{2}<2^{64}$ для некоторого $t \in\{2, m\}$, то взятие по модулю можно выполнять в $t$ раз реже.

Еще одно замечание следует сделать относительно емкостной сложности приведенного метода умножения. При $N<2^{32}$ каждый элемент ДПФ хранится в одном 32-битном машинном слове, в то время как один коэффициент двоичного многочлена представим с помощью 1 бита. Это приводит к тому, что для хранения матриц ДПФ необходимо в 32 раза больше оперативной памяти, чем для хранения исходных матриц-множителей и результата. Тем не менее, требуемый объем памяти можно существенно сократить за счет проведения вычислений в определенном порядке. Например, можно поступать следующим образом.

- Вычислить матрицу ДПФ $\hat{A}$.

- Для каждого столбца $B_{j}^{\downarrow}$ матрицы $B$ :

- вычислить столбец векторов ДПФ $\hat{B}_{j}^{\downarrow}$;

- для каждой строки $\vec{A}_{i}$ матрицы $\hat{A}$ вычислить произведение $\hat{c}_{i j}=\vec{A}_{i} \hat{B}_{j}^{\downarrow}$, а для него - обратное ДПФ, получив элемент результата $c_{i j}$.

В этом случае необходимо хранить целиком только одну матрицу векторов ДПФ матрицу $\hat{A}$, а также один столбец векторов ДПФ $\left(\hat{B}_{j}^{\downarrow}\right)$ и один вектор ДПФ результата $\hat{c}_{i j}$. При больших размерах матриц-множителей данный подход позволяет сократить требуемый объем памяти почти в 3 раза.

\section{5. Результаты экспериментов}

Как отмечалось выше, алгоритм на основе БПФ дает преимущество перед алгоритмом Карацубы лишь при умножении многочленов достаточно большой степени. Алгоритм умножения многочленов ограниченной степени, приведенный в разделе 3 , был реализован автором на языке $\mathrm{C}++$ для проведения экспериментального сравнения его эффективности с алгоритмом Карацубы. В качестве реализации алгоритма Карацубы была использована функция умножения двоичных многочленов известной библиотеки NTL версии 5.3 (данная функция использует в общем случае алгоритм Карацубы, а для многочленов маленькой степени - специфические функции, приводящие к сокращению трудоемкости).

В табл. 1 приведено сравнение времени умножения двух многочленов степени $2^{k-1}-1$ с помощью функции библиотеки NTL $\left(T_{\mathrm{NTL}}\right)$ и с помощью авторской реализации изложенного алгоритма $\left(T_{2}\right)$. Для алгоритма на основе БПФ приведено также время вычисления ДПФ для одного многочлена и время перемножения двух векторов ДПФ. Постановка экспериментов выполнялась на ЭВМ с процессором Intel XEON 2,53 ГГц под OC Windows 7 , для компиляции программного кода использовался 64-разрядный компилятор среды разработки Microsoft Visual Studio 2008.

Замечание 1. Определенное несоответствие роста трудоемкости расчетной формуле объясняется нюансами реализации и оптимизации алгоритмов, а также характером работы оперативной памяти.

Из таблицы видно, что авторская реализация алгоритма на основе БПФ работает быстрее функции библиотеки NTL лишь при умножении многочленов очень больших 
Таблица 1. Сравнение времени работы алгоритма Карацубы (реализация NTL) и алгоритма из раздела 3 на основе БПФ (реализация автора)

\begin{tabular}{c||c|c||c|c}
$k$ & $T_{\mathrm{NTL}}, \mathrm{мc}$ & $T_{2}$, мс & одно БПФ, мс & умножение ДПФ, мс \\
\hline \hline 22 & 1280 & 3225 & 1070 & 15 \\
23 & 3850 & 5911 & 1960 & 31 \\
24 & 11650 & 14192 & 4710 & 62 \\
25 & 35181 & 30806 & 10227 & 125 \\
26 & 103889 & 60463 & 20071 & 250
\end{tabular}

Таблица 2. Сравнение алгоритмов умножения двоичных полиномиальных матриц

\begin{tabular}{c|c|c|c}
$m \times m$ & $k$ & $T_{\mathrm{NTL}}, \mathrm{Mc}$ & $T_{2}, \mathrm{Mc}$ \\
\hline $2 \times 2$ & 18 & 140 & 422 \\
$4 \times 4$ & 18 & 1030 & 1528 \\
$8 \times 8$ & 18 & 8081 & 6099 \\
$16 \times 16$ & 18 & 65318 & 26536 \\
$16 \times 16$ & 14 & 842 & 1201 \\
$32 \times 32$ & 14 & 6583 & 5992 \\
$64 \times 64$ & 14 & 52120 & 24757 \\
$128 \times 128$ & 14 & 420 & 126 \\
$256 \times 256$ & 14 & 3412 & 736
\end{tabular}

степеней. Это связано с тем, что для выполнения умножения двух многочленов необходимо выполнить три БПФ; перемножение же векторов ДПФ выполняется во много раз быстрее. Следовательно, можно получить существенный выигрыш, если каждый многочлен будет участвовать в большом количестве умножений, что характерно, например, для умножения полиномиальных матриц.

Как было отмечено в предыдущем параграфе, при перемножении полиномиальных матриц можно использовать БПФ, вычислив сначала матрицы ДПФ каждого элемента множителей, затем перемножив полученные матрицы ДПФ и, наконец, выполнив обратное ДПФ для получения результата. Если перемножаются полиномиальные матрицы размера $m \times m$, то каждый элемент (многочлен) участвует в $m$ произведениях. Следовательно, трудоемкость умножения полиномиальных матриц размера $m \times m$ степени $n$ с помощью БПФ будет $O\left(m^{2} n \log n+m^{3} n\right)$ операций в поле.

В табл. 2 приведены результаты экспериментов по сравнению времени умножения полиномиальных матриц размера $m \times m$ степени $2^{k-1}-1$ стандартным способом $\left(T_{\mathrm{NTL}}\right)$ и с помощью БПФ $\left(T_{2}\right)$. В первом случае матрицы перемножались обычным способом с использованием функции библиотеки NTL для умножения многочленов-элементов матриц. Во втором случае применялся метод на основе БПФ, описанный в разделе 4 , с авторской реализацией БПФ и стандартным умножением матриц ДПФ.

Видно, что даже на сравнительно небольших размерах и степени полиномиальных матриц алгоритм на основе БПФ работает существенно быстрее. Заметим, что для нахождения решений системы линейных уравнений при факторизации 768-битного числа авторами работы [3] был использован алгоритм, одним из этапов которого было умножение двух полиномиальных матриц размера $1536 \times 1536$ и степени $565000 \approx 2^{19}$. 


\section{6. Заключение}

В работе рассмотрены вопросы, связанные с реализацией умножения многочленов и полиномиальных матриц на ЭВМ. Для двоичных многочленов ограниченной степени предложен эффективный метод реализации умножения с помощью БПФ, использующий в качестве базовых операций сложение и умножение машинных слов по модулю $2^{64}$. Проведен ряд экспериментов по сравнению времени работы реализации предложенного метода с функцией умножения двоичных многочленов, реализованной в известной библиотеке NTL. Показано, что использование предложенного метода при умножении двоичных полиномиальных матриц дает существенный выигрыш по сравнению с алгоритмом Карацубы, реализованным в библиотеке NTL, даже при сравнительно небольших размерах и степени матриц.

\section{Список литературы}

1. Thomé E., Subquadratic computation of vector generating polynomials and improvement of the block Wiedemann algorithm. J. Symb. Comput. (2002) 33, 757-775.

2. Coppersmith D., Solving linear equations over $G F(2)$ via block Wiedemann algorithm. Math. Comput. (1994) 62, №205, 333-350.

3. Kleinjung T., Aoki K., Franke J., Lenstra A. K., Thomé E., Bos J. W., Gaudry P., Kruppa A., Montgomery P. L., Osvik D. A., te Riele H., Timofeev A., Zimmermann P., Factorization of a 768-bit RSA modulus. Lecture Notes Comput. Sci. (2010) 6223, 333-350.

4. Карацуба А. А., Сложность вычислений. Труды Математического института им. В. А. Стеклова РАН (1995) 211, 186-202.

5. Schönhage A., Strassen V. , Schnelle Multiplikation grosser Zahlen. Computing (1971) 7, 281-292.

6. Burgisser P., Clausen A., Shokrollahi A., Algebraic complexity theory. Springer, Berlin, 1997.

7. Furer M. , Faster integer multiplication. In: Proc. STOC'2007. ACM, New York, 2007, pp. 57-66.

8. Кнут. Д. Э., Искусство программирования: Получисленные методы, 2. Вильямс, Москва, 2007.

9. Pollard J. M., The fast Fourier transform in a finite field. Math. Comput. 1971, 365-374.

10. Schönhage A., Schnelle Multiplikation von Polynomen über Körpern der Charakteristik 2. Acta Inf. (1977) 7, 395-398.

Статья поступила 15.02.2012. 